Florian Aschinger, Alexandra Becker, Nicole Gageur und Hannes Weichert

\title{
Forschungsfeld Lernwelt Hochschule
}

\author{
Methodische Zugänge zur Analyse einer differenzierten \\ Struktur
}

\section{Ausgangslage}

Ausgangspunkt des Forschungsprojekts Lernwelt Hochschule ist die Darstellung des Ist-Zustands der deutschen Hochschullandschaft unter einer studierendenorientierten Perspektive. Dieser bislang nur selten gewählte Forschungszugang beruht

auf dem hochschuldidaktischen Verständnis des Bologna-Prozesses, der Kompetenzen als Lernergebnisse und didaktische Arrangements zur Förderung des ,student engagements“ betont [...] (Wildt/Wildt 2011, 11, H.i. O.).

Betrachtet man die Entwicklungen der deutschen Hochschullandschaft unter dieser Perspektive, lassen sich vielfältige Herausforderungen konstatieren.

Die Umorientierung in der Lehre zu einer stärkeren Lernendenorientierung und die Weiterqualifizierungen der Lehrenden führen dazu, dass das Thema Lehre unter den beteiligten Akteurinnen und Akteuren intensiver diskutiert wird. Durch diesen Austausch untereinander werden soziale Aushandlungsprozesse in Gang gesetzt, die schrittweise einen Kulturwandel im Sinne der dargestellten Strategie anstoßen und fördern (Knutzen et al. 2016, 296).

Bislang wird im Zusammenhang der Diskussion über die studierendenorientierte Hochschule fast ausschließlich die Hochschullehre in den Blick genommen. Da zu einer studierendenorientierten Hochschule jedoch mehr gehört als eine veränderte Hochschuldidaktik, wird im Projekt Lernwelt Hochschule versucht, die Perspektive Studierendenorientierung auf die weiteren Dimensionen der Hochschulentwicklung auszuweiten. Dies schlägt sich zum Beispiel im Servicegedanken oder der Einbindung der Studierenden in die Entscheidungsbildungsprozesse der Organisation Hochschule, in der Anpassung der physischen Lehr- und Lernräume zur Realisierung der kompetenzorientieren Lehre oder auch im Hinblick auf die Optimierung von studierendenrelevanten Prozessen in der Gestaltung der digitalen Strukturen einer Hochschule nieder. 
Da es im Rahmen des Projektes Lernwelt Hochschule vor allem darum geht, die Situation von Hochschulen bezogen auf einen studierendenorientierten $\mathrm{Zu}$ gang genauer in den Blick zu nehmen und damit das Verstehen, Interpretieren und Beschreiben der Phänomene im Vordergrund steht, wurde eine hermeneutisch-interpretative Forschungsperspektive eingenommen und ein induktives Vorgehen angelegt.

Dabei wird berücksichtigt, dass sich die einzelnen Hochschulen unterschiedlich entwickelt haben und je nach angestrebter oder bereits bestehender Typisierung (Hochschulart, Trägerschaft und Standort) unterschiedlichen Rahmenbedingungen unterworfen sind. Es wird vor allem darauf geachtet, wie tradierte Strukturen das Bild der Hochschulen heute prägen. So hat Luckmann grundsätzlich darauf hingewiesen, dass die konstruierte institutionelle Ordnung - wie sie auch in Hochschulen zu finden ist - intergenerativ als selbstverständliche „natürliche“ Umwelt tradiert wird, ohne dass jedoch kritisch hinterfragt wird, woher bestimmte erlernte Handlungsabläufe oder objektiv geglaubtes Wissen herrühren (Luckmann 2006, 84-85). Es geht also auch darum, scheinbar feststehende Strukturen kritisch $\mathrm{zu}$ hinterfragen und im Modus der Studierendenorientierung zu reflektieren.

Um vor diesem Hintergrund die Lernwelt Hochschule genauer zu betrachten, wurde ein konsekutives Vorgehen gewählt (siehe Abbildung 1): Zunächst wurden Akteurinnen und Akteure aller staatlich zugelassenen Hochschulen Deutschlands eingeladen, an einer Online-Befragung teilzunehmen. Darauf aufbauend wurden Leitfadeninterviews mit Akteurinnen und Akteuren aus Hochschulen geführt, bei denen die Auswertung auf Good Practice schließen ließ. Im Rahmen von Fallstudien wurde die Perspektive von Studierenden eingebunden. Interviews mit internationalen Expertinnen und Experten öffneten den Blick auf internationale Veränderungsprozesse. Ein weiterer wichtiger Aspekt des Projektes ist die Analyse strukturbildender Hochschuldokumente wie Zielvereinbarungen, Entwicklungs- und Strukturpläne, Landeshochschulgesetze, etc. 
Aufbau der Erhebung - Stichproben

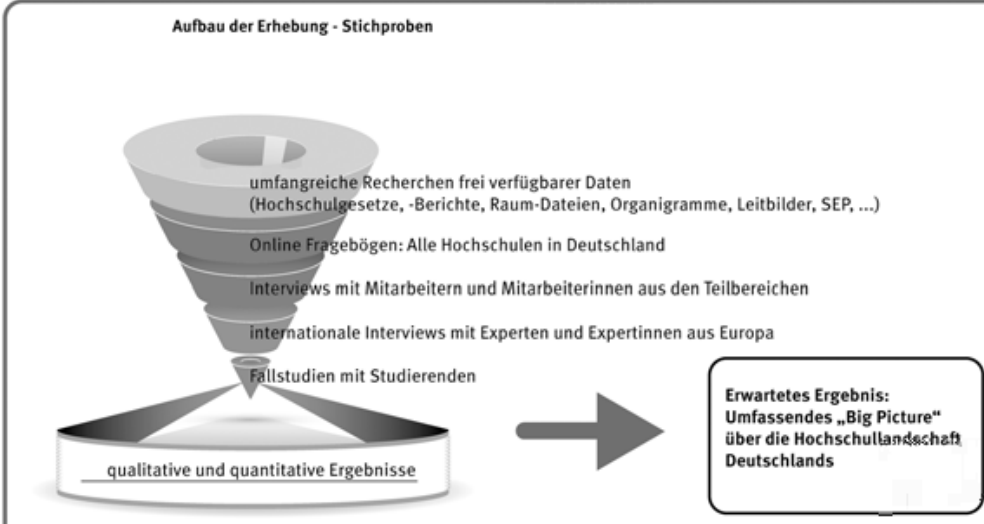

Abb. 1: Aufbau der Erhebungsstruktur (eigene Darstellung)

Um das Vorgehen in seiner Gesamtheit nochmals deutlicher zu machen, werden die Abläufe kurz dargestellt. Zunächst wurden Akteurinnen und Akteure (Hochschulleitungen, Bibliotheksleitungen, Leitungen von IT-Abteilungen und Leitungen von Infrastruktur-Abteilungen) aller Hochschulen Deutschlands eingeladen, an der Online-Befragung teilzunehmen. Die Hochschulen, die sich im Rahmen der Befragung identifizierten - 178 nahmen diese Möglichkeit wahr -, bildeten den Pool für die Stichprobe der Leitfadeninterviews. Anschließend wurden die Ergebnisse der vier Fragebögen mittels eines Good Practice-Kriterienkataloges ${ }^{1}$ sortiert und - um diese Fragebögen, da sie über eine unterschiedliche Anzahl an Fragen und unterschiedlicher Anzahl erreichbarer Good Practice-Punkte verfügen, vergleichbar zu machen - prozentual umgerechnet.

So wurde zum Beispiel im Fragebogen nach Sondermitteln für die Weiterentwicklung der Lehre gefragt. Diese Frage konnte mit einer Mehrfachnennung beantwortet werden: „Drittmittel“, „Mittel aus der Universität/Hochschule“, „Länderspezifische Sonderprogramme“, „Maßnahmen zur Eigenfinanzierung“ und „Gibt es nicht“. Da im Normalfall mehr verfügbare Mittel zur Unterstützung der Weiterentwicklung der Lehre beitragen, wurde bei dieser Frage für jede Antwort, außer für „Gibt es nicht“, ein Bonuspunkt vergeben. Hier konnten also im Idealfall vier Punkte erreicht werden. Zunächst wurde für jeden der vier Fragebögen eine maximale Punktzahl ermittelt. Diese entspricht 100 Prozent Good Practice-Punkte in dem Ranking in der jeweiligen Fragebogenart. Hatte eine

1 Wird auf der Projekthomepage publiziert: www.zukunftlernwelthochschule.de. 
Hochschule zum Beispiel die Hälfte der maximalen Punkte erreicht, so hatte sie 50 Prozent im Ranking.

Auf der Basis dieses Ranking wurden schließlich 29 Interviews geführt. Diese wurden um 12 Interviews ergänzt, die auf der Basis einer Literaturrecherche aus Hochschulen mit Good Practice herausgefiltert wurden. Als besonders interessant erachtete Fälle der Hochschulen, in denen Interviews stattfanden, wurden zur Teilnahme an den Fallstudien eingeladen.

Ergänzend wurden internationale Expertinnen und Experten in Bezug auf Hochschulentwicklung gebeten, sich für ein Interview zur Verfügung zu stellen, um deren Einschätzung über die Trends der Hochschulentwicklung im europäischen Ausland und bezogen auf die Entwicklung der Hochschullandschaft Deutschlands in die Auswertung einbeziehen zu können.

Für die Analyse der Hochschuldokumente wurden die frei verfügbaren Entwicklungs- und Strukturpläne der staatlichen Hochschulen sowie deren Zielvereinbarungen mit den Bundesländern eingesehen. Zudem wurden private Hochschulen aller Hochschularten mit der Bitte angeschrieben, entsprechende Strategiedokumente aus ihren Institutionen für das Forschungsprojekt zur Verfügung zu stellen.

Insgesamt gelang es so, ein umfassendes, wenn auch nicht vollständiges Bild der deutschen Hochschullandschaft $\mathrm{zu}$ erhalten. Im Folgenden wird das methodische Vorgehen der verschiedenen Erhebungsschritte vorgestellt.

\section{Online-Befragung}

Im Zentrum des Projektes Lernwelt Hochschule steht die Online-Befragung aller Hochschulen Deutschlands. Um die Grundgesamtheit zu erreichen, wurden die Kontaktmöglichkeiten bei der Hochschulrektorenkonferenz (HRK)2, diversen Verteiler der Bibliotheksverbände ${ }^{3}$ und des Netzwerkes Deutsche Initiative Netzwerkinformation e. V. (DINI) genutzt und im Internet recherchiert.

Die Online-Befragung erfasst grundlegende Informationen zu den vier Dimensionen (Hochschulorganisation, Hochschuldidaktik, digitale Strukturen und physische Lehr- und Lernräume), um einen Überblick der Selbstwahrnehmung der Hochschulen zu erhalten. Sie ist hypothesengenerierend angelegt. Dies ist dem Desiderat in diesem Forschungsgebiet geschuldet, dass es bislang keine Studien gibt, die unter einer studierendenorientierten Perspektive die

2 https://www.hochschulkompass.de/hochschulen/downloads.html.

3 Unter anderem aus der Sektion 4 „Wissenschaftliche Spezialbibliotheken“ des Deutschen Bibliotheksverbandes. 
Hochschullandschaft in Deutschland umfassend durchleuchten. In diesem ersten Schritt sollte so das Erkunden von möglichen Annahmen induktiv ermöglicht werden.

Da es in Deutschland über 500 staatlich zugelassene Hochschulen gibt, wäre der Erhebungsaufwand durch persönliche Interviews mit Akteurinnen und Akteuren zu groß gewesen. So bot sich eine Online-Umfrage an. „OnlineBefragungen sind zeitlich und räumlich unabhängig“ (Wagner/Hering 2014, 662, H. i. O.). Weitere Vorteile dieser Methode

liegen in der Möglichkeit, Multimedia-Elemente, automatisierte Filterführungen, Plausibilitäts-Checks der Eingaben sowie Randomisierungen der Antwortmöglichkeiten oder Frageblöcke zu verwenden [...] [sowie] in dem schnellen Rücklauf (Fühles-Ubach 2013, 115).

Die Nachteile sind darin zu sehen, dass keine Kontrolle darüber besteht, wer schlussendlich den Fragebogen ausfüllt (Zerr 2003, 20) und dass keine Möglichkeit zur persönlichen Interaktion besteht, sodass den Begleittexten zu den Fragen eine hohe Bedeutung zukommt.

Auch lässt sich nicht ohne weiteres feststellen, wie aufmerksam die Befragten den Fragebogen bearbeiten. Zusätzlich besteht bei langen Befragungen die Gefahr einer hohen Abbruchquote während des Ausfüllens. In diesem Zusammenhang gilt auch, dass der Item-Nonresponse, also die Nichtbeantwortung einzelner Fragen, bei Online-Erhebungen oftmals um einiges höher ausfällt als bei anderen Methoden (Zindler/Pohl 2015, 305).

Die Befürchtung, dass diese Befragung zu lang sein könnte, zeigte sich beim Pretest bestätigt, an dem 46 Personen aus den vier verschiedenen Zielgruppen teilnahmen. Scholl verweist auf die Bedeutung des Pretests:

Bei der Fragebogenkonstruktion sollten nicht nur Pretests durchgeführt werden, um das Verständnis bestimmter Wörter, Sätze oder Instruktionen durch die Befragten zu erkennen, sondern diese Pretests können experimentell eingesetzt werden, um die Wirkung unterschiedlicher Formulierungen zu testen. (Scholl 2013, 93)

Der Pretest zeigte, dass der 82 Fragen umfassende Gesamtfragebogen, der nicht in voller Tiefe von einer Person, ohne dass sie Rücksprache mit den anderen Bereichen hätte halten müssen, beantwortet werden konnte und dadurch die vollständige Beantwortung zu zeitaufwändig gewesen wäre. Als Ergebnis des Pretests wurde der Ausgangsfragebogen inhaltlich nach den Dimensionen Hochschulorganisation, Hochschuldidaktik, digitale Strukturen und physische Lehr- und Lernräume geordnet in vier Fragebögen untergliedert. Dies bedeutete, dass die Befragten nicht nur einen geringeren Zeitaufwand hatten, sondern diesen auch ohne Nach- und Rückfragen ausfüllen konnten, was die Nutzungs- 
freundlichkeit deutlich erhöhte. Dabei wurden folgende Organisationsbereiche für die Beantwortung des jeweiligen Fragebogens eingeladen: Hochschulleitung, Bibliotheksleitung, Leitung der IT-Abteilung und Leitung der Infrastrukturabteilung. Hier zeigte sich, dass nicht von allen Hochschulen Kontaktdaten $\mathrm{zu}$ den Infrastruktur- und IT-Abteilungen auffindbar waren, sodass hier nicht alle Hochschulen angeschrieben werden konnten und bei diesen Zielgruppen ein geringerer Rücklauf zu verzeichnen ist.

In der Laufzeit von Dezember 2017 bis zum November 2018 gingen insgesamt 562 gültige Antwortfragebögen ein. Davon entfielen 34 Prozent auf Hochschulleitungen, 41 Prozent auf Bibliotheksleitungen, 15 Prozent auf Leitungen der IT-Abteilungen und zehn Prozent auf Leitungen der Infrastrukturabteilungen.

Grundsätzlich empfiehlt Faulbaum zur Erhöhung der Rücklaufquote; „sofern die finanziellen Mittel dafür vorhanden sind, die Durchführung von Nachfassaktionen“ (Faulbaum 2011, 166). Diese Erinnerung wurde - bedingt durch die einjährige Laufzeit des Fragebogens - zweimal (03.2018 und 10.2018) versendet.

Auch die Formulierung der Fragen ist von zentraler Bedeutung, da die Komplexität eines Fragetextes ein Problem für die Antwortqualität darstellt, wenn man bedenkt, dass mit der Komplexität des Textes oft eine entsprechende Komplexität der kognitiven Leistung einhergeht, die erbracht werden muss, um den Text zu verstehen (Faulbaum 2011, 241). Unklare Formulierungen können zur Folge haben, dass die intendierte Bedeutung der Forschenden sich deutlich von dem unterscheidet, was der bzw. die Befragte darunter versteht. Tourangeau et al. verweisen auf das Problem:

The danger in vagueness is supposed to be that some respondents will choose one way to make a vague question precise, whereas others will choose a different way, leading to uninterpretable variability in the responses. (Tourangeau et al. 2000, 45)

Auch wenn Eindeutigkeit in der Sprache sicherlich ein schwer zu realisierendes Ziel ist, so wird doch deutlich, dass der präzisen Frageformulierung ein hoher Stellenwert zukommt, und hier für die Güte und Qualität, insbesondere für die Validität der Befragungsdaten eine der größten Herausforderungen zu sehen ist, denn die Validität zeigt, ob das Messinstrument auch das abbildet, was erfasst werden sollte (Schnell et al. 2011, 146).

In der Online-Befragung wurden offene Fragen vermieden, da diese zeitaufwändig zu beantworten sind (Bortz/Döring 2007, 254). Dazu wurden vor allem folgt man der Einteilung von Petersen $(2014,87)$ - Fakten-, Wissens- und Einstellungsfragen gestellt, und Motiv- und Verhaltensfragen vermieden, da diese 
zum einen nicht dem Forschungsziel dienten und zum anderen weitere mögliche Fehlerquellen dargestellt hätten.

Ausgewertet wurde mittels deskriptiver Statistik. Es wurden Häufigkeitsanalysen für die Gesamtheit und für die gefundenen Typen (Hochschulart, Hochschulträgerschaft und Bundesland) gerechnet. Im Zuge dieser Auswertung wurden Typen gebildet. Kluge gliedert die Ziele der Typenbildung in

rein deskriptive zur Strukturierung eines Untersuchungsbereiches [...] und in heuristische und theoriebildende Funktionen, um inhaltliche Sinnzusammenhänge zwischen und innerhalb der Typen zu analysieren (Kluge 1999, 43).

Die in dieser Auswertung gebildeten Typen bezogen sich auf die Merkmale Bundesland, Hochschulart und Trägerschaft. Hierbei handelt es sich um Realtypen. Diese

stellen [...] eine Kombination von Merkmalen dar, die im Gegensatz zum Idealtypus empirisch nachweisbar, d. h. in der Realität vorhanden und vorfindbar ist (Reinhold 1991, 469).

Auch wurde eine Assoziationsanalyse zur „Prüfung von Zusammenhängen zwischen verschiedenen Variablen eingesetzt“ (Hartmann 2015, 161). Das Chi-Quadrat vergleicht „die Besetzung [der Kreuztabelle] mit einer Besetzung, die man erwarten würde, wenn keine Beziehung zwischen den Variablen bestünde“ (Benninghaus 2007, 104). Oftmals waren in dieser Erhebung die theoretischen Häufigkeiten zu gering, sodass die Monte Carlo-Methode, „ein numerischer Algorithmus, der aus einer Wahrscheinlichkeitsverteilung eine Zufallszahl ermittelt“ (Diaz-Bone/Weischer 2014, 253), zum Resampling, „eine Technik, aus einer bereits vorliegenden Stichprobe mehrere Stichproben wiederholt zu ziehen“ (Diaz-Bone/Weischer 2014, 351), genutzt werden musste, um eine Aussage über den Zusammenhang zwischen Typ und Item treffen zu können.

Der Abgleich der Stichprobe mit der Grundgesamtheit (alle staatlich zugelassenen Hochschulen Deutschlands) zeigte sich problembehaftet. Es ist nicht möglich, eine übereinstimmende Sicht mittels der genutzten Quellen ${ }^{4}$ auf die Grundgesamtheit deutscher Hochschulen zu bekommen. Die Angaben unterscheiden sich in unterschiedlichen Ausmaßen. Zunächst fällt auf, dass sich die Anzahl der jeweils zugrunde liegenden Gesamtangaben unterscheidet. Das Destatis-Portal des Bundes nennt eine Anzahl von 429 staatlich anerkannten

\footnotetext{
4 Als zugrundeliegende Quellen wurden: Die Liste der Hochschulen der Hochschulrektorenkonferenz im Hochschulkompass (https://www.hochschulkompass.de/hochschulen/downloads.html), Bundesamt für Statistik (https://www.destatis.de/DE/Themen/Gesellschaft-Umwelt/Bildung-Forschung-Kultur/Hochschulen/Tabellen/hochschulen-hochschularten.html) und der statistischen Ämter der Länder des Bundes genutzt.
} 
Hochschulen, die Angaben der statistischen Ämter der Bundesländer ergeben summiert eine Anzahl der Hochschulstandorte von 586, der Hochschulkompass der Hochschulrektorenkonferenz (HRK) weist eine Anzahl von 395 aus und die telefonische Nachfrage bei Destatis ergab eine Gesamtanzahl von 471 für die privaten und öffentlich-rechtlichen Hochschulen im Jahr 2017. Destatis merkt dazu an:

Der Berichtskreis der Hochschulfinanzstatistik deckt sich in der Regel mit dem Berichtskreis der übrigen Hochschulstatistiken (Studierenden-, Prüfungs- und Personalstatistiken). Allerdings lassen sich auch hier aus erhebungstechnischen und haushaltswirtschaftlichen Gründen gewisse Abweichungen nicht vermeiden. Die Ausgaben neugegründeter Hochschulen werden häufig noch nicht im Gründungsjahr, sondern erst in den Folgejahren in die Hochschulfinanzstatistik einbezogen. (Statistisches Bundesamt 2019, 9)

Damit ist es nicht möglich, eine valide Aussage über die Anzahl der Hochschulen in Deutschland zu treffen. Dies mag bei der HRK dem Umstand geschuldet sein, dass sie nur Daten ihrer Mitglieder nutzt oder erhebt. Unklar bleibt, warum die Daten, aufgeschlüsselt nach den Kategorien Standort, Hochschultyp und Trägerschaft bei Destatis nur über die Hochschulfinanzstatistik zu erlangen waren, und warum sich die Angaben zwischen Bundesländern und dem Portal des Bundes unterscheiden. Selbst unter der Berücksichtigung, dass die Länder die Anzahl der Standorte angaben, und es 115 (resp. 158) Standorte geben mag, denen Hochschulen zugeordnet werden könnten, ist die Diskrepanz zwischen den Angaben nicht zu klären, zumal nicht zu eruieren war, wie viele Standorte von welchen Hochschulen gezählt wurden.

Aus diesem Umstand resultieren auch die Unterschiede in den Kategorien. Auffallend in der Verteilung nach Bundesländern ist, dass Zahlen, welche das jeweilige statistische Landesamt angab, von denen der Hochschulfinanzstatistik und den Angaben der HRK abweicht. So gibt es zum Beispiel, wenn man den Landesämtern folgt, in Mecklenburg-Vorpommern sieben Hochschulstandorte, die der Hochschulfinanzstatistik zufolge zu neun Hochschulen gehören. Die HRK nennt sechs Hochschulen in diesem Bundesland. Da sich die Anzahlen der zugrunde gelegten Gesamtanzahl unterscheiden, verwundert es nicht, dass sich auch die Anteile der Hochschultypen unterscheiden. So variieren die Anteile der Universitäten zwischen 25 Prozent bei den Angaben des Destatis-Portals bis zu 30 Prozent bei den Angaben der Hochschulfinanzstatistik. Hinzu kommt die unterschiedliche Zuordnung der Hochschulen zu den Typen. So bildet die HRK einen eigenen Typ, in dem verschiedene Hochschulen zusammengefasst werden, es werden Theologische Hochschulen als Typ ausgewiesen, wobei unklar bleibt, ob diese Klasse auf der Trägerschaft oder der inhaltlichen Ausrichtung 
beruht. Die hier nur kurz skizzierte Problemlage weist bereits auf ein Desiderat von Hochschulforschung hin.

Den Überblick über die Verteilung der Trägerschaften zu erhalten, ist ebenfalls erschwert, da nicht in allen Angaben die gleiche Kategorisierung zugrunde gelegt wurde; die Statistischen Ämter der Bundesländer unterscheiden lediglich in öffentlich-rechtliche und private Trägerschaft, die HRK und die Hochschulfinanzstatistik in öffentlich-rechtliche, private und kirchliche Trägerschaft. Unterstellt man nun, dass die statistischen Ämter der Länder die kirchlichen Hochschulen der Kategorie „Privat“ zuschlagen, so zeigt sich, dass die Anteile der öffentlich-rechtlich getragenen Hochschulen einen Anteil von 61 Prozent bei der HRK bis zu 66 Prozent bei den Angaben der Hochschulfinanzstatistik einnehmen.

Die quantitative Online-Befragung ist einer der Bausteine, mit dem die Hochschullandschaft Deutschlands beschrieben werden kann. Diese Erhebung liefert grundlegendes Faktenwissen, was mit Hilfe von Interviews sicherlich nur schwer abzubilden gewesen wäre. Des Weiteren bilden die Ergebnisse in Verbindung mit dem Good Practice-Ranking eine gute Basis, um die Good Practice der Hochschullandschaft Deutschlands herauszuarbeiten. Wie gezeigt, gibt es Forschungslücken, die es in weiteren Schritten zu schließen gilt, um einen systematisierten und strukturierten Überblick zu gewinnen. Die Ergebnisse finden sich in Becker/Stang 2020.

\section{Leitfadeninterviews mit Hochschulakteurinnen und -akteuren}

Zur Durchführung der Leitfadeninterviews wurden analog zur Online-Befragung vier verschiedene Versionen des Leitfadens für Hochschulleitungen, Bibliotheksleitungen, Leitungen der IT-Abteilung und Leitungen der Infrastrukturabteilung formuliert. Diese beinhalteten jeweils auf die Aufgabenbereiche der Hochschulakteurinnen und -akteure zugeschnittene Fragen. Die Ausarbeitung und Gliederung der Fragen geschah dabei unter Zuhilfenahme von Beispielleitfäden für Experteninterviews (Gläser/Laudel 2006), und die Entwicklung der Frageninhalte erfolgte auf der Basis der vier Dimensionen der Lernwelt Hochschule: (1) Hochschulorganisation, (2) Hochschuldidaktik, (3) digitale Strukturen und (4) physische Lehr- und Lernräume. Dabei sind die Bereiche digitale Strukturen, physische Lehr- und Lernräume und Hochschuldidaktik im Rahmen der Hochschulorganisation eng miteinander verknüpft, um eine im Idealfall 
umfassende, hochschulweite Gestaltung zu erreichen (NMC 2018; P21 2018; Radcliffe 2009). Den vier Dimensionen wurden in der Literatur recherchierte Good Practice-Kriterien (u. a. DINI 2013; JISC 2015; UCISA 2016) zugeordnet, welche in Abgleich mit den in der Online-Befragung behandelten Themen zur Bestimmung der Befragungsinhalte dienten.

Die Gliederung der Leitfäden sah jeweils eine für den Gesprächsbeginn einfach formulierte Einstiegsfrage zum aktuellen Entwicklungsstand an der Hochschule vor. So wurde bei den Hochschulleitungen nach aktuellen Aufgaben im Rahmen der Hochschulstrategie, bei den Leitungen der IT-Abteilungen nach dem Stand der Digitalisierung und bei den Bibliotheksleitungen und den Leitungen der Infrastrukturabteilungen nach der Ausstattung und den Funktionen der vorhandenen Lernräume gefragt. Darauf folgten themenspezifische Frageblöcke, welche aus einer oder mehreren Hauptfragen sowie optionalen Nachfragen bestanden. Hier enthielt der Leitfaden für die Hochschulleitungen Fragen nach Strategien und Maßnahmen, insbesondere zur Entwicklung einer studierendenorientierten Hochschule sowie zu digitalem Lehren und Lernen. Bei den Bibliotheksleitungen stand die Konzeption, Ausstattung und Nutzung physischer Lernräume in der Bibliothek im Vordergrund. Bei den Leitungen der Infrastrukturabteilungen lag der Fokus ebenso auf den physischen Lernräumen, wobei hier Lehr- und Lernräume an der gesamten Hochschule thematisiert wurden. Für die Leitungen der IT-Abteilungen wurden Fragen zu vorhandenen digitalen Strukturen und zur technischen Ausstattung der (digitalen) Lernräume formuliert. Am Ende gab es zwei Abschlussfragen: Zum einen wurde um eine Einschätzung gebeten, wie die Lernwelt Hochschule nach Ansicht der Befragten zukünftig aussehen wird; zum anderen wurde nach Wünschen an das Projekt Lernwelt Hochschule gefragt, um Möglichkeiten der Unterstützung der Hochschulen bei der Lernweltgestaltung zu erfassen. Zur Prüfung der Leitfäden wurden insgesamt fünf Probeinterviews geführt, welche dazu dienten, den Ablauf der Interviews zu testen und zu Anpassungen in den Frageformulierungen führten.

Für den ersten Kontakt mit den potentiellen Teilnehmenden wurde ein EMail-Anschreiben für jede der vier Befragtengruppen formuliert, welches die wichtigsten Informationen zum Projekt und zum Ablauf der Interviews enthielt. Für Personen, welche noch nicht an der Online-Befragung teilgenommen hatten, wurde im Anschreiben zusätzlich eine Einladung zur Online-Befragung eingefügt. Diesem E-Mail-Anschreiben wurden zur Erhöhung der Transparenz eine verkürzte Version des jeweiligen Leitfadens sowie eine Einwilligungserklärung zur Aufnahme und Verarbeitung der Interviews angehängt. Die verkürzte Leitfadenversion bot den Teilnehmenden die Möglichkeit, Einblick in die im Interview zu besprechenden Themen zu gewinnen und sich auf das Interview vorzu- 
bereiten. Die Einwilligungserklärung diente zur Aufklärung über den Umgang mit den Interviewtranskripten und enthielt die Erlaubnis zur Aufzeichnung und Weiterverarbeitung der Interviews.

Für die Zusammenstellung der Stichprobe wurden zunächst die Ergebnisse der Online-Befragung anhand von Good Practice-Kriterien gewichtet, um die einzelnen Fälle in eine Rangordnung zu bringen und so die am besten bewerteten Fälle auszuwählen. Da für die Rekrutierung der Interviewpartnerinnen und -partner die Angabe des Hochschulnamens in der Online-Befragung erforderlich war, konnten nicht alle Fälle zur Stichprobengenerierung herangezogen werden. Am Ende bildeten 178 Fälle die Gesamtheit der hier möglichen Interviewpartnerinnen und -partner, wobei ein Good Practice-Wert von 60 Prozent in den Antworten nicht unterschritten werden sollte. Hinzu kamen Empfehlungen möglicher Interviewpartnerinnen und -partner auf der Basis von Recherchen und aus der Projektgruppe. Bei diesen Empfehlungen handelte es sich um Hochschulen und auch Einzelpersonen, welche in einer oder mehreren der vier Dimensionen der Lernwelt Hochschule einen sehr guten Ansatz verfolgen. Die finale Einsatzstichprobe (inkl. Nachrückerinnen und Nachrücker) enthielt insgesamt 67 Fälle, darunter 29 Hochschulleitungen, 14 Bibliotheksleitungen, sechs Leitungen von Infrastrukturabteilungen und 18 Leitungen einer IT-Abteilung (siehe Tabelle 1).

Tab. 1: Verteilung der Befragtengruppen.

\begin{tabular}{llcc}
\hline Zugehörigkeit Hochschulakteur/in & $\begin{array}{l}\text { Good Practice } \\
\text { Onlinebefragung }\end{array}$ & Empfehlungen & Gesamt \\
\hline Hochschulleitung & 18 & 11 & $\mathbf{2 9}$ \\
Bibliotheksleitung & 9 & 5 & 14 \\
Leitung Infrastrukturabteilung & 1 & 5 & 6 \\
Leitung IT-Abteilung & 17 & 1 & 18 \\
Gesamt & 45 & 22 & 67 \\
\hline
\end{tabular}

Die niedrige Einsatzstichprobe bei der Leitung Infrastrukturabteilung ist bedingt durch die ebenfalls relativ kleine Einsatzstichprobe und die geringe Beteiligung in der Online-Befragung. Weiterhin enthält die Einsatzstichprobe fünf Fälle für Probeinterviews, welche aufgrund ihrer inhaltlichen Qualität in die Auswertung mit aufgenommen wurden. Um zu gewährleisten, dass es sich bei der ausgewählten Stichprobe um eine möglichst heterogene Gruppe handelte, wurden diese hinsichtlich der Kriterien Hochschulart, Trägerschaft und Bundesland kontrolliert.

Zur Rekrutierung wurde die Einsatzstichprobe unter Berücksichtigung der Standorte der zu interviewenden Personen auf die Projektmitarbeiterinnen und 
-mitarbeiter an den Projektstandorten Hamburg, Stuttgart und Bamberg aufgeteilt. Die ausgewählten Personen wurden zunächst per E-Mail angeschrieben und nach ca. einer Woche telefonisch kontaktiert oder zuerst angerufen, um dann gegebenenfalls die Einladung per E-Mail zu schicken. In einigen Fällen waren mehrere Kontaktversuche notwendig, um die Teilnahme zu klären. Insbesondere Hochschulleitungen waren schwer erreichbar; teilweise waren keine direkten Kontaktdaten vorhanden, sodass die Kontaktaufnahme über Dritte erfolgte; oftmals wurde eine baldige Rückmeldung zugesagt, welche aber nicht erfolgte, sodass diese Fälle als „Absage“ gewertet wurden. Die Kontaktierungsversuche wurden in einer separaten Tabelle pro Interviewerin oder Interviewer dokumentiert. Sofern Absagen erfolgten, wurden mittels der Good PracticeRangliste Nachrückerinnen und Nachrücker bestimmt und je nach Auslastung und Standort der zu kontaktierenden Person einer Interviewerin oder einem Interviewer zugeordnet.

Die Interviews wurden vor Ort durchgeführt. Vor Beginn des Interviews erfolgte jeweils die Einwilligung der Interviewpartnerinnen oder -partner zur Aufzeichnung und Weiterverarbeitung der Daten. Nach dem Start der Aufnahme gab es eine kurze Einleitung durch die Interviewerin oder den Interviewer, in welcher die Projektziele sowie die konkrete Zielsetzung des Interviews erläutert wurden. Im Anschluss daran konnten gegebenenfalls weitere Fragen zum Projekt und zum Interview geklärt werden. Darauf folgte dann das Interview anhand des Leitfadens. Bei manchen Interviews, insbesondere mit den Bibliotheksleitungen, gab es zudem die Möglichkeit, die Räumlichkeiten der Hochschule zu besichtigen. Dies lieferte wertvolle Eindrücke, welche als Zusatzinformationen bei der Auswertung der Interviews dienten.

Letztlich konnten von den insgesamt 67 kontaktierten Personen 41 Zusagen eingeholt werden. Tabelle 2 gibt Aufschluss über die realisierte Stichprobe.

Tab. 2: Verteilung der kontaktierten Personen.

\begin{tabular}{llcl}
\hline Zugehörigkeit Hochschulakteur/in & Zusagen & Absagen & Gesamt \\
\hline Hochschulleitung & 13 & 17 & $\mathbf{3 0}$ \\
Bibliotheksleitung & 13 & 1 & $\mathbf{1 4}$ \\
Leitung Infrastrukturabteilung & 4 & 2 & 6 \\
Leitung IT-Abteilung & 11 & 6 & $\mathbf{1 7}$ \\
Gesamt & $\mathbf{4 1}$ & $\mathbf{2 6}$ & $\mathbf{6 7}$ \\
\hline
\end{tabular}

Insgesamt haben 61 Prozent der angefragten Personen einem Interview zugestimmt und teilgenommen. Dabei zeigt sich bei den Bibliotheksleitungen eine besonders hohe Teilnahmebereitschaft. Die Verteilung der 41 Zusagen nach 
Hochschulart, Trägerschaft und Bundesland zeigt eine zufriedenstellende Heterogenität der Befragtengruppe, welche größtenteils der Verteilung der Einsatzstichprobe entspricht.

Die Transkription der Interviews erfolgte anhand vorab erstellter Transkriptionsregeln mit der Textanalysesoftware MAXQDA, welche auch zur Auswertung der Interviews diente. Neben Regelungen zur Vereinheitlichung des Formats und der Sprache sowie zum Umgang mit schwer oder gar nicht verständlichen Gesprächsteilen, wurde in diesem Arbeitsschritt auch eine erste Anonymisierung der Interviews umgesetzt: Um keine direkten Rückschlüsse auf die befragten Personen oder deren Einrichtungen ziehen zu können, wurden alle Eigennamen entfernt. Für die Auswertung der Interviews wichtige Informationen (z. B. Position der Befragten an der Hochschule) wurden separat gespeichert.

Die Auswertung der Texte orientierte sich an der Methode der qualitativen Inhaltsanalyse nach Mayring (Kuckartz 2007; Mayring 2009, 2015). Dabei bot sich ein deduktiv-induktives Vorgehen an, bei welchem die Kategorienbildung als iterativer Prozess erfolgte (Rädiker/Kuckartz 2019): Die Transkripte wurden zunächst mittels eines deduktiv anhand der Interviewleitfäden erstellten Kategoriensystems kodiert (,konzeptgesteuerte Kategorienbildung“; Rädiker/Kuckartz 2019, Kap. 8.3) - für jeden Leitfaden (und dementsprechend für jede der vier Befragtengruppen) gab es zunächst jeweils ein Kategoriensystem. Für Textstellen, die nicht zugeordnet werden konnten, wurden induktiv neue Kategorien gebildet („datengesteuerte Kategorienbildung“; Rädiker/Kuckartz 2019, Kap. 8.4). Darauf erfolgte eine Feinkodierung des Materials, um eine „Überladung“ einzelner Kategorien sowie die Sammlung mehrerer Themen in einer Kategorie $\mathrm{zu}$ vermeiden. Hierbei wurden möglichst trennscharfe Unterkategorien gebildet. Mehrfachkodierungen waren $\mathrm{zu}$ vermeiden, begründete Ausnahmen aber erlaubt. Nachdem alle Interviews kodiert waren, wurde das Kategoriensystem überarbeitet und vereinheitlicht, sodass die Unterteilung in die vier Befragtengruppen entfiel und nur noch inhaltlich relevante Kategorien vorhanden waren. Gleiche oder inhaltlich sehr ähnliche Kategorien wurden dabei zusammengeführt. Am Ende gab es ein Kategoriensystem für alle Interviews.

Nach Abschluss der Kategorisierung wurden die in den einzelnen Kategorien enthaltenen Textstellen hinsichtlich Gemeinsamkeiten und Besonderheiten untersucht. So konnten einzelne Themenbereiche zusammengefasst und die wichtigsten Aspekte zur Beschreibung der Gestaltung von Lernräumen an deutschen Hochschulen herausgefiltert werden. Beispielsweise konnten so alle Textstellen, in welchen die Beteiligung der Studierenden an der Entwicklung von Konzepten zur Lernraumgestaltung thematisiert wird, gesammelt analysiert werden. Weiterhin wurden zur Analyse der kategorisierten Interviews Variablen 
gebildet, welche die Strukturierung des Materials nach relevanten Merkmalen wie Hochschulart, Trägerschaft oder Studierendenzahl und somit gruppenspezifische Analysen ermöglichten. Da die durchgeführten Interviews als ,informatorische Interviews“ zu sehen sind, die „der deskriptiven Erfassung von Tatsachen aus den Wissensbeständen der Befragten“ (Lamnek 2005, 333) dienen, gestaltet sich die Analyse - entsprechend dem informatorischen Charakter der Interviews - stark beschreibend und weniger theorieorientiert. Die Ergebnisse finden sich in Aschinger 2020.

\section{Ethnografische Fallstudien}

Während sich die Online-Befragung und die Leitfadeninterviews mit den Akteurinnen und Akteuren an die Leitungs- und Planungspersonen der Hochschulen richteten, wurde der Blick bei den ethnografischen Fallstudien auf die Perspektive der Studierenden gerichtet (siehe Abbildung 2). Der Fokus liegt auf den Prozessen und den Erfahrungen aus studentischer Sicht, der „Student Experience" ${ }^{\text {. }}$.

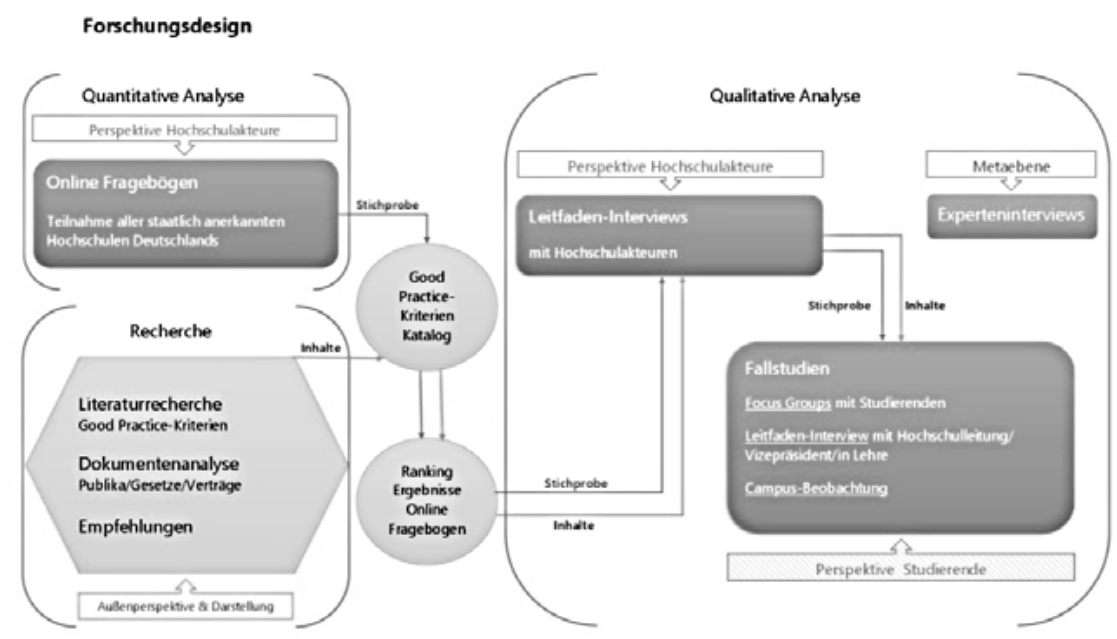

Abb. 2: Forschungsdesign - Überblick über die Methodenauswahl (eigene Darstellung).

5 Der im anglo-amerikanischen Bereich geprägte Begriff der „Student Experience“ umfasst das studentische Erleben an den Hochschulen in ganzheitlicher und erweiterter Sicht, das neben Lehr- und Lernstrukturen auch soziale und kulturelle Aspekte beinhaltet. 
Die Ergebnisse der Fallstudien sollen Hinweise liefern auf Zusammenhänge zwischen der Hochschulstrategie zur Gestaltung der Hochschulangebote wie Hochschulorganisation und dem Service in Bezug auf Hochschullehre, physischer sowie digitaler Lernraum und dem studentischen Erleben der Lernwelt Hochschule. Im Sinne der Methodentriangulation kommen den ethnografischen Fallstudien im Rahmen des Projekts Lernwelt Hochschule wesentliche Aufgaben zu: Illustration und Erfassung der Perspektive der Studierenden und damit derjenigen, die die Angebote konkret nutzen. Mit Hilfe der ethnografischen Fallstudien soll gezeigt werden, welche typischen Abläufe, Prozesse oder Angebote an der Hochschule besonders erfolgreich sind oder in welchen Bereichen es Probleme gibt beziehungsweise die Studierenden unzufrieden sind. Sie ermöglichen den bewussten Perspektivwechsel und zielen darauf, mehr über die Erfahrungen, Bewertungen und Bedürfnisse der Studierenden zu erfahren. Mit der begrenzten Anzahl von fünf Fallstudien haben die Ergebnisse einen eher exemplarischen Charakter, liefern allerdings interessante Einblicke in den Hochschulalltag von Studierenden.

Im Sinne eines ganzheitlichen Ansatzes wird zunächst die Hochschulleitung für die Fallstudien separat interviewt. Leitendes Thema dabei ist die Strategie der Hochschule, für die Studierenden eine effektive und angenehme Lernwelt zu gestalten. Wie die Strategie von den Studierenden wahr- und angenommen wird, soll dann die Fokusgruppendiskussion mit den Studierenden zeigen. Im Rahmen der Beobachtung erkunden die Forschenden den zu untersuchenden Campus und dokumentierten ihre Eindrücke fotografisch und schriftlich. Zusammengefasst lauten die Forschungsfragen der ethnografischen Fallstudien: Welches Selbstverständnis hat die Hochschule hinsichtlich strategischer Planung und Umsetzung für die eigene Lernwelt entwickelt, welche tatsächlichen Erfahrungen haben die Studierenden mit diesen Angeboten gemacht und wie werden diese Angebote genutzt? Die ethnografische Forschung ermöglicht Einsichten in den Kontext der Lernaktivitäten von Studierenden. Es geht darum, wie Studierende ihre Aufgaben bewältigen und wie sie die Lernwelt Hochschule erleben. Die drei gewählten ethnografischen Methoden der Fallstudien gewähren drei unterschiedliche Zugänge zur Lernwelt Hochschule und ermöglichen den Vergleich von Planung und Nutzung, von Strategie und studentischem Erleben.

Die Fragen für die Leitfäden, einerseits für das Interview mit der Hochschulleitung, andererseits für die Gruppendiskussion mit den Studierenden, wurden mit Hilfe des SPS²-Verfahrens (Helfferich 2011, 182-185; Kruse 2015, 230-235) generiert. Entsprechend wurden in einer Brainstorming-Phase Fragen geSammelt, danach wurden sie Sortiert, dann gePrüft, schließlich wurden Fragen geStrichen und zusammengelegt und zum Schluss wurden die gebildeten Cluster 
unter Fragen, die Erzählungen evozieren sollen, Subsummiert $\left(=\mathrm{S}^{2} \mathrm{PS} \mathrm{S}^{2}\right)$. Da feststand, dass die Fallstudien von verschiedenen Personen aus dem Projekt durchgeführt werden, wurde bei allen drei Methoden der Fallstudie jeweils ein Leitfaden eingesetzt, um eine Vergleichbarkeit und Konsistenz zu gewährleisten. Durch die Leitfäden wird sichergestellt, dass unterschiedliche Frageformulierungen nicht das Ergebnis beeinflussen und dadurch bei der Interpretation der Antworten berücksichtigt werden müssten. Im Folgenden werden die einzelnen Erhebungsschritte der Fallstudien näher erläutert.

\section{Leitfadeninterview mit der Hochschulleitung}

Das Leitfadeninterview mit der Hochschulleitung fokussiert ausdrücklich auf die Bedürfnisse der Studierenden. Die im Rahmen der Fallstudie relevanten Ansprechpartnerinnen und -partner aus der Hochschulleitung sind für die Lehre zuständige Personen, da sie die Studierenden am besten im Blick haben (Vizepräsident/in bzw. Prorektor/in Lehre und/oder Studium). Die Fragen korrespondieren inhaltlich mit denen der Gruppendiskussion. Zu prüfen ist die Fragestellung, wie die strategischen Planungen zur Lernwelt Hochschule in der Umsetzung von den Studierenden erfahren werden. Der Aufbau des Leitfadens orientiert sich an dem, der bereits für die Leitfadeninterviews mit den Akteurinnen und Akteuren entwickelt wurde. Der durchgeführte Pretest hat keine wesentlichen Änderungen an dem entwickelten Leitfaden ergeben.

\section{Gruppendiskussion (Focus Groups) mit den Studierenden}

Bei der Planung und Konzeption der Gruppendiskussion wurden die Kriterien für einen guten Diskussionsleitfaden berücksichtigt, nämlich dass:

- er eine Unterhaltung herbeiführt, in dem die Teilnehmenden ermutigt werden an der Diskussion teilzunehmen,

- er den Soziolekt der Teilnehmenden benutzt,

- die Fragen der/dem Moderator/in leicht über die Lippen kommen,

- die Fragen kurz und klar formuliert sind (lange Fragen können die Antwortenden verwirren).

- offene Fragen gestellt werden - die Fragen verlangen nach Erklärungen, Beschreibungen und genaueren Ausführungen. (Krueger 2009, 36-37; Helfferich 2011, 102-108; Kruse 2015, 215-218) 
Außerdem sollen die Teilnehmenden aufgefordert werden, sich an persönliche Erlebnisse zu erinnern (Lamnek 2005, 100). Wichtig ist zu prüfen, ob die Fragen wirklich offen für das Fremde sind oder aus dem Relevanzsystem der Forschenden kommen. Nach Helfferich und Kruse sollten bei der Entwicklung des Leitfadens Suggestivfragen, Präsuppositionen, geschlossene Fragen und Mehrfachfragen vermieden werden. Ebenfalls sollten keine wertenden Fragen, keine Warum-Fragen und keine Scham- oder Schuldgefühle auslösenden Fragen gestellt werden (Helfferich 2011, 102-108; Kruse 2015, 215-218).

Für die reine Gruppendiskussion mit den Studierenden wurden zwei Stunden veranschlagt, für das Moderationsteam mit Vor- und Nachbereitung drei bis vier Stunden (Aufbau, Abbau, Postskript). Das Moderationsteam sorgt in Absprache mit den Hochschulen für Getränke, Snacks, Namensschilder und Stifte. Incentives sind nicht geplant. Die Gruppendiskussion wird mit einem Tonaufnahmegerät mit zwei Mikrofonen aufgenommen. Notizen helfen zu erkennen, wer spricht und teilweise die Mimik und Gestik der Teilnehmenden zu erfassen. Außerdem wurde ein Postskript nach der Diskussion von dem Projektteam ausgefüllt (Lamnek 2005, 169-173). Es beinhaltet Notizen zu der (Gesprächs-)Atmosphäre, den Befindlichkeiten der Teilnehmenden beziehungsweise der Forschenden, (Gesprächs-)Verlauf, Interaktionen, Besonderheiten, auffallende Themen und gegebenenfalls Störungen (Kruse 2012, 278).

An der Gruppendiskussion nahmen jeweils ca. fünf Studierende teil. Es handelt sich dabei insofern um homogene Gruppen, als dass sie aus Studierenden ein- und derselben Hochschule gebildet werden, die sich idealerweise in einem höheren Semester befinden. Die Gruppe ist künstlich zusammengesetzt, da die Studierenden sich vorab nicht zwangsläufig kennen und sich freiwillig auf einen Aufruf zur Gruppendiskussion melden.

Der Aufbau einer Gruppendiskussion gliedert sich grundsätzlich in Eröffnungsfragen/Eisbrecherfragen (opening questions), Hinführungsfragen (introductory questions), Überleitungsfragen (transistion questions), Schlüsselfragen (key questions) und Schlussfragen (ending questions) (Lamnek 2005, 96-104; Krueger 2009, 38-40). Hieran orientiert sich auch die Struktur des Leitfadens für die Gruppendiskussion:

\section{Warming up/ Smalltalk}

- Wie geht es dir heute?

- Wie hast du hergefunden?

- Heute ist das Wetter prima, nicht? 
Begrüßung

- Vorstellung des Projekts

- Einwilligungserklärung unterschreiben lassen

- Informationsblatt zum Projekt und Datenschutz verteilen bzw. auslegen

- Start Aufnahme -

Eisbrecher/Vorstellungsrunde (opening questions)

- Die Eröffnungsfrage ist leicht und kurz zu beantworten, beispielsweise nach Name, Studienfach und Hobby. ${ }^{6}$

Themenblöcke 1-X

- Stimulus/ Grundreiz: Photo Survey, Szenario, offene Fragen

- Fragen zu inhaltlichen Aspekten, Aufrechterhaltungsfragen und konkrete Nachfragen

Schlussfragen

- Ende Aufnahme -

Verabschiedung und Dank

Als Gesprächsanreiz für Themenblock 1 diente ein kleiner Photo-Survey. Die teilnehmenden Studierenden wurden vorab gebeten, zwei Fotos zu machen: „Fotografiere bitte deinen Lieblingslernort auf dem Campus und den Platz in der Hochschule, an dem du dich verlassen fühlst“. Sowohl die Idee als auch die Fragenformulierung sind der Studie von Foster und Gibbons Studying Students entlehnt (Foster/Gibbons 2007, 40-47). In der Praxis haben sich gerade diese beiden Formulierungen als besonders ergiebig erwiesen. Der Fotoauftrag ist bewusst einerseits offen, andererseits die Gefühlsebene ansprechend gewählt. Es soll gerade nicht intellektualisiert und reflektiert werden. Die Studierenden erläutern dann beim ersten thematischen Schwerpunkt Physischer Lehr- und Lernraum ihre Fotos und sollen darüber miteinander ins Gespräch kommen. So unterstützen die Fotos die Eröffnung der Gruppendiskussion. Rekrutierung, Fragen, Aufbau und Länge der Gruppendiskussion wurden durch einen Pretest geprüft und entsprechende Anpassungen vorgenommen. So wurde zum Bei-

6 Alle Teilnehmenden sollen die Eröffnungsfrage zu Beginn der Fokusgruppensitzung beantworten, denn je länger ein/e Teilnehmer/in schweigt, umso schwieriger ist es, sie oder ihn zum Sprechen zu bewegen. Die Eröffnungsfrage wird normalerweise nicht analysiert, sie dient dazu, sich bekannt zu machen und für ein angenehmes Diskussionsklima zu sorgen. 
spiel die Anzahl der Teilnehmenden von ursprünglich bis zu zehn geplanten auf maximal fünf Teilnehmende gesenkt, da einerseits der Rücklauf ohne Incentives relativ gering war und andererseits die Gruppendiskussion sonst zu lange gedauert hätte.

\section{Beobachtung/Campusbegehung}

Die Campusbegehung findet als wissenschaftliche, teil-strukturierte, nicht-teilnehmende, offene Feldbeobachtung statt (Döring/Bortz 2016, 323-330; Lamnek/Krell 2016, 515-531). Im Fokus der Beobachtung steht nicht das Verhalten der Studierenden, sondern die Attraktivität des Campus und wie bei dessen Gestaltung auf die Bedürfnisse der Studierenden eingegangen wird. Hier interessieren die Studierenden als Indikator: Ein Ort, an dem sich viele Studierende aufhalten, ist ein wichtiger Ort; wie nutzen die Studierenden die Angebote, werden sie eventuell zweckentfremdet? Angestrebt ist eine neutrale Beobachtung mit unabhängigem, möglichst objektivem Blick. Die Beobachtungen wurden zum Teil mit Fotos dokumentiert. Aus datenschutzrechtlichen Gründen waren meistens keine Fotos mit Studierenden möglich.

Grundsätzlich interessieren folgende Beobachtungen auf dem Campus:

- Wo halten sich besonders viele Studierende auf beziehungsweise wo lernen die Studierenden?

- Wie finden sich Studierende zurecht? Gegebenenfalls wird dies durch Fotografien des Leitsystems oder durch „verlorene, suchende“ Studierende dokumentiert.

Außerdem liegt der Fokus auf der Ausstattung und Verteilung von

- Seminarräumen,

- Hörsälen,

- Lernzentren,

- Medienzentren,

- Bibliotheken,

- Cafés,

- Automaten mit Getränken und/oder Snacks,

- Mensen,

- Servicestellen.

Auffälligkeiten oder Nicht-Vorhandensein waren in einem Beobachtungsprotokoll zu dokumentieren. Die Fotos werden anhand der qualitativen Inhaltsanaly- 
se nach Mayring (Mayring 2009; Mayring 2015) und Kuckartz (Kuckartz 2016) mit MAXQDA analysiert.

Die fünf Fallstudien wurden an ausgewählten Hochschulen durchgeführt. Auswahlkriterien waren Teilnahme an der Online-Befragung und den Leitfadeninterviews mit den Hochschulakteurinnen und -akteuren, wobei die Hochschulen nach Rücklauf sortiert und nach Good Practice-Kriterien gerankt waren. Außerdem spielten Hochschulmerkmale (z. B. Hochschultyp und -art) eine Rolle, die Art der Mitbestimmung der Studierenden und die geografische Verteilung. Zudem sollten Duale Hochschulen/Berufsakademien vertreten sein.

Die drei Elemente der jeweiligen Fallstudie wurden jeweils von einem Projektteam bestehend aus zwei Personen durchgeführt, wobei eine Person durchgängig an allen fünf Fallstudien teilnahm, um eine größtmögliche Kontinuität zu gewährleisten. Pro Hochschulort wurden ein bis zwei Tage Aufenthalt eingeplant. Die ausgewählten Hochschulen wurden angeschrieben und zur Teilnahme an den Fallstudien eingeladen. Bei Zusagen wurde das weitere Vorgehen gemeinsam geplant und abgestimmt. Die Hochschulen wurden um Mithilfe gebeten, um über die Studierendenverteiler die Studierenden zur Gruppendiskussionen einzuladen. Datenschutz- und Persönlichkeitsrechte wurden berücksichtigt.

Folgendes Material gehörte zur Standardausstattung: Fotoapparat, Smartphone, sehr gutes Aufnahmegerät mit mindestens zwei Mikrophonen, Ausdrucke des Photo-Surveys, Leitfadenfragen, Zettel für Notizen, Vordrucke zum Protokollieren, Einverständniserklärungen für Interview und Gruppendiskussion, Informationsblatt mit Projektvorstellung, Getränke und Snacks für die Gruppendiskussion, Karton und Stifte für Namensschilder.

Die Transkription der Audiodateien erfolgte überwiegend durch studentische Hilfskräfte. Es wurden einheitliche Transkriptionsregeln (die gleichen wie schon bei den Leitfadeninterviews mit den Hochschulakteurinnen und -akteuren) sowohl für die Interviews als auch die Gruppendiskussion verwendet. Die Transkription erfolgte direkt mit MAXQDA. Die Auswertung des Materials wurde entsprechend mit Hilfe der Textanalysesoftware MAXQDA durchgeführt. Das Kategoriensystem wurde sowohl deduktiv vorab mittels Good Practice-Kriterien und Themen aus der Online-Befragung und den vorab geführten Leitfadeninterviews als auch induktiv direkt aus dem Material erstellt. Die Ergebnisse finden sich in Gläser/Kobsch 2020. 


\section{Dokumentenanalyse}

Mit der Online-Befragung sowie den Leitfadeninterviews mit ausgewählten Hochschulakteurinnen und -akteuren und den Fallstudien an ausgewählten Good Practice-Institutionen sollte die Realität der Lernweltgestaltung in der deutschen Hochschullandschaft erfasst werden. Um einen Abgleich der auf diese Weise erzielten Ergebnisse mit den offiziellen strategischen Zielvorstellungen der untersuchten Hochschulen vorzunehmen, richtete sich der Blick im Rahmen der Dokumentenanalyse auch auf zentrale strategische Dokumente. Um zudem die Perspektive der Träger der staatlichen Hochschulen in diesen Abgleich einzubeziehen, wurden die Landeshochschulgesetze dahingehend analysiert, ob in ihnen gesetzliche Regelungen zu den vier Untersuchungsdimensionen vorgenommen werden.

Zur Gewährleistung der Vergleichbarkeit des Primärquellenmaterials in historischer Hinsicht wurden ausschließlich Dokumente und Gesetze in die Untersuchung einbezogen, die im Jahr 2017 gültig waren. Als zentrale strategische Hochschuldokumente wurden Entwicklungs- und Strukturpläne (ESPs) sowie im Fall der staatlichen Hochschulen deren Zielvereinbarungen mit den jeweiligen Bundesländern identifiziert, da es sich bei diesen beiden Dokumententypen um von den zuständigen Gremien offiziell beschlossene, für die Gesamtheit der Hochschulen sowohl verbindliche als auch grundlegende Schriftstücke handelt. Leitbilder wurden zwar recherchiert, jedoch nach Durchsicht als in den meisten Fällen allgemein gehaltene pointierte Formulierungen des Selbstverständnisses bewertet und daher in der Analyse nicht berücksichtigt.

Im Zuge der Recherche der Dokumente wurden alle frei verfügbaren bundesdeutschen Hochschul-ESPs, Hochschul-Zielvereinbarungen, Hochschul-Leitbilder und Landeshochschulgesetze eruiert und nach Hochschulart und -trägerschaft strukturiert zusammengestellt. Dabei stellte sich heraus, dass nur eine geringe Anzahl an ESPs privater Hochschulen frei verfügbar vorlag. Deshalb wurden die Leitungen 76 privater Hochschulen, deren entsprechende Dokumente nicht öffentlich zugänglich waren, mit der Bitte angeschrieben, ihre ESPs für das Forschungsprojekt zur Verfügung zu stellen. Die Quantität des Primärquellenmaterials dieser Sub-Untersuchungsgruppe änderte sich auf diese Weise nicht. Da auch bei kirchlichen Hochschulen und Verwaltungshochschulen keine ausreichende Masse an ESPs ermittelt werden konnte, um diese als verlässliche Basis für valide Aussagen heranzuziehen, setzt sich das Primärquellenmaterial der Hochschuldokumente aus den Zielvereinbarungen der 14 Bundesländer, die diese frei zur Verfügung stellen, sowie den zum Stichzeitpunkt aktuellen ESPs staatlicher Hochschulen aller Typen - mit Ausnahme der Verwaltungs- 
hochschulen - zusammen. Hinzu kommen die Landeshochschulgesetze der 16 Bundesländer sowie drei Landeskunsthochschul- und fünf Landesberufsakademiegesetze.

Die Auswertung der ESPs und Zielvereinbarungen erfolgt anhand eines an den vier Untersuchungsdimensionen des Forschungsprojekts ausgerichteten Kategoriensystems (Meyen et al. 2019, 150-154). Um entsprechende Strukturen des Materials abzubilden, werden Textabschnitte, die den gebildeten Kategorien entsprechen, systematisch herausgezogen, gegebenenfalls paraphrasiert, auf typische Ausprägungen untersucht und schließlich pro Kategorie zusammengefasst sowie auf Häufigkeiten analysiert (Mayring 2009, 496-473; Mayring 2015, 13, 50, 71, 97). In einem iterativen Prozess werden im Verlauf der Untersuchung die Kategorien und das Kategoriensystem immer wieder überprüft und gegebenenfalls einer Revision unterzogen. Dabei werden in einem ersten Arbeitsschritt alle Dokumente auf Textstellen zu den vier Untersuchungsdimensionen durchgesehen. Diese Grobkategorisierung wird anschließend einer Subkategorisierung in inhaltliche Detail-Cluster unterzogen. Alle Subkategorien werden daraufhin auf potentielle Doppelungen oder inhaltliche Überschneidungen kontrolliert und gegebenenfalls zusammengefasst. Sich im Verlauf des Kodierungsprozesses ergebende Mehrfachkodierungen aus den Untersuchungsdimensionen Hochschuldidaktik und digitale Strukturen werden in der überwiegenden Zahl der Fälle aufgelöst und die entsprechenden Textstellen derjenigen der beiden Dimensionen mit der größeren inhaltlichen Deckung zugeordnet. Mit Beendigung der Kategorisierungstätigkeit werden die Ergebnisse sowohl auf hervorstechende Ausprägungen als auch auf Übereinstimmungen und sich bei diesen potentiell ergebenden Häufigkeiten überprüft. Die auf diese Weise generierten thematischen Sparten werden jeweils entlang der Kriterien Bundesland, Hochschulart und Untersuchungsdimension strukturiert zusammengestellt, um die Voraussetzung für an diesen Kriterien orientierte und vergleichende Analyseschritte zu schaffen.

Die Landeshochschulgesetze, in der jeweils zum Untersuchungszeitpunkt geltenden Fassung, werden nicht einer dezidierten juristischen Analyse unterzogen, sondern unter dem Gesichtspunkt der vier Untersuchungsdimensionen des Forschungsprojekts Lernwelt Hochschule dokumentarisch aufbereitet. Dementsprechend werden die Landeshochschulgesetze der 16 Bundesländer, die Kunsthochschulgesetze der Länder Nordrhein-Westfalen und Saarland sowie die Berufsakademiegesetze der Länder Hamburg, Niedersachsen, Saarland, Sachsen und Schleswig-Holstein (KMK 2019) auf Bestimmungen zu Hochschuldidaktik, digitalen Strukturen und physischen Lehr und Lernräumen durchgesehen. Die Untersuchungsdimension der Hochschulorganisation wird im Zusammenhang der Landeshochschulgesetze nicht im Sinne der allgemeinen Hochschul- 
organisation, sondern in demjenigen der Organisation der Hochschule als Lernwelt und der Organisation der Hochschule als studierendenorientierte Institution verstanden. Die Resultate der Durchsicht der Hochschulgesetzgebung werden zum einen thematisch entlang der vier Untersuchungsdimensionen und zum anderen unter dem Aspekt der Trägerschaft für jedes der 16 Bundesländer zusammengestellt. Diese Zusammenstellungen sollen dem Zweck dienen, den übrigen Erhebungs- und Analyseschritten des Forschungsprojekts eine Basis für einen Abgleich mit den gesetzlichen Vorgaben der jeweiligen Hochschulträger zu bieten. Die Ergebnisse finden sich in Weichert 2020a und Weichert $2020 b$.

\section{Interviews mit internationalen Expertinnen und Experten}

Die Durchführung von Interviews mit internationalen Expertinnen und Experten auf dem Gebiet der Hochschul-Lernwelten zielt darauf ab, die Arbeit des Forschungsprojekts Lernwelt Hochschule vor dem Hintergrund internationaler Forschungsergebnisse zu reflektieren sowie die eigene Forschungstätigkeit über die Kooperation mit den Expertinnen und Experten in den internationalen akademischen Diskurs einzubringen.

Als Grundlage der Interviews wurde ein Leitfaden-Fragebogen zur qualitativen Befragung konzipiert (Werner 2013, 130-134), dessen thematische Grobgliederung in die Aspekte Erfahrungen und Good Practice, Blick auf Deutschland und Blick in die Zukunft auf Basis aktueller Schwerpunkte der Fachliteratur sowie Forschungsschwerpunkte der Projektpartnerinnen und -partner erstellt wurde. Unter Orientierung an Leitfaden- (Helfferich 2014, 565-568) und Experteninterviewmodellen (Helfferich 2014, 570-573) wurden anschließend Detailfragen $\mathrm{zu}$ den drei thematischen Blöcken entwickelt. In einem ersten Probe-Interview stellten sich die dabei entstandenen Fragen des mit dem Blick auf die deutsche Hochschullandschaft befassten Themenblocks als zu spezifisch heraus. Deshalb wurden alternativ 22 Thesen zur aktuellen deutschen Hochschullandschaft aus den Ergebnissen der Online-Befragung und der Interviews mit Hochschulakteurinnen und -akteuren generiert. In den Interviews wurden dann die jeweiligen Personen um Stellungnahme zu einer speziell für sie getroffenen Auswahl an Thesen gebeten.

Um den internationalen Expertinnen und Experten eine Einordnung sowohl der Interviewanfrage als auch des Forschungsprojekts zu ermöglichen, wurde 
eine Kurzzusammenfassung erster Projektergebnisse erstellt. Diese Kurzzusammenfassung, der in Bezug auf den auf deutsche Hochschulen ausgerichteten Themenblock individualisierte Fragebogen-Leitfaden sowie ein am Standard der Universität Edinburgh orientierter Interview Consent Form (University of Edinburgh 2013) wurden ins Englische übersetzt. Gemeinsam mit dem zum Gespräch einladenden Anschreiben bildeten diese Dokumente das Material, das den potentiellen Interviewpartnerinnen und -partnern im Sinne eines transparenten Gesprächsprozesses vorab zur Verfügung gestellt wurde.

Die Auswahl der internationalen Fachleute erfolgte mittels Empfehlungen aus der Projektlenkungsgruppe sowie unter Hinzuziehung der Expertise externer Hochschul-Consultants. Mit dieser Auswahl sollten in der Lernweltgestaltung aktive Hochschulen, nationale und internationale Hochschulzusammenschlüsse oder -verbände sowie die internationale Hochschulberatung abgebildet werden. Die so ausgewählten vier zu interviewenden Personen aus vier verschiedenen Ländern wurden im Erstkontakt per E-Mail angeschrieben und circa eine Woche später telefonisch adressiert. Die Durchführung der Interviews ging im ersten Halbjahr 2019 vonstatten, oblag drei Projektmitarbeiterinnen und -mitarbeitern und konnte nach individueller Absprache der Beteiligten im direkten Gespräch vor Ort, via Telefonie oder Video-Telefonie vollzogen werden. In den einzelnen Gesprächen waren nach einer Einleitung in das Forschungsprojekt und während des Interviews beiderseitige Nachfragen möglich.

Eine erste Transkription der Audioaufnahmen der Interviews durch studentische Hilfskräfte wurde von den Projektbeteiligten, die das Gespräch geführt hatten, gegengelesen. Die Auswertung der Transkripte erfolgte aufgrund der Heterogenität des Materials nicht anhand eines Analysemodells, sondern als Gegenüberstellung und Vergleich der inhaltlichen Gemeinsamkeiten und Unterschiede entlang der drei Themenblöcke beziehungsweise der Einzelfragen. Als Besonderheit der Auswertung gilt es zu beachten, dass zwei der Interviews auf Englisch und zwei auf Deutsch geführt wurden. Bei der Analyse muss somit der Vergleichbarkeit und den Unterschieden der verwendeten zweisprachigen Terminologie erhöhte Bedeutung und Aufmerksamkeit zukommen (Schittenhelm 2017, 109-112). Die Ergebnisse finden sich in Weichert/Stang 2020.

\section{Fazit}

Betrachtet man die methodischen Zugänge im Projekt Lernwelt Hochschule, wird deren multiperspektivische Struktur deutlich. Um die methodische Komplexität des Projektes anschaulich zu machen, wurde bewusst ein eigenständi- 
ges Methodenkapitel ohne Bezug zu den Ergebnissen erstellt. Den Autorinnen und Autoren ist es ein Anliegen, diese Struktur darzulegen, um Anregungen für Forschungsvorhaben im Kontext der Hochschul- und Bildungsforschung zu geben, die versuchen, einen ganzheitlichen Blick auf Bildungsinstitutionen zu werfen. Um die Möglichkeiten und Grenzen von Veränderungsprozessen wissenschaftlich auszuloten, erscheint ein solches Vorgehen geboten.

\section{Literatur}

Aschinger, F. (2020): Konzeption und Management der Lernwelt Hochschule - Herausforderungen und Good Practice aus Sicht der Hochschulakteurinnen und -akteure. In: A. Becker; R. Stang (Hrsg.): Lernwelt Hochschule. Dimensionen eines Bildungsbereichs im Umbruch. Berlin; Boston: De Gruyter Saur, 124-150.

Becker, A.; Stang, R. (2020): Lernwelt Hochschule im Aufbruch. Zentrale Ergebnisse einer Befragung. In: A. Becker; R. Stang (Hrsg.): Lernwelt Hochschule. Dimensionen eines Bildungsbereichs im Umbruch. Berlin; Boston: De Gruyter Saur, 71-123.

Benninghaus, H. (2007): Deskriptive Statistik. Eine Einführung für Sozialwissenschaftler. Wiesbaden: Springer.

Berger, P.; Luckmann, T. (1980): Die gesellschaftliche Konstruktion der Wirklichkeit. Frankfurt/ M: Fischer.

Bortz, J.; Döring, N. (2007): Forschungsmethoden und Evaluation für Human -und Sozialwissenschaftler. Wiesbaden: Springer.

Diaz-Bone, R.; Weischer, C. (2014): Methodenlexikon für die Sozialwissenschaften. Wiesbaden: Springer.

DINI - Deutsche Initiative für Netzwerkinformation e. V. (2013): Die Hochschule zum Lernraum entwickeln. Kassel: Kassel University Press.

Döring, N.; Bortz, J. (2016): Forschungsmethoden und Evaluation in den Sozial- und Humanwissenschaften. Berlin; Heidelberg: Springer.

Eberle, T. S. (2000): Lebensweltanalyse und Handlungstheorie. Beiträge zur verstehenden Soziologie. Konstanz: UVK.

Faulbaum, F. (2011): Methodische Grundlagen der Umfrageforschung. Wiesbaden: Springer.

Foster, N. F.; Gibbons, S. (2007): Studying Students. The Undergraduate Research Project at the University of Rochester. Chicago: Association of College and Research Libraries.

Fühles-Ubach, S. (2013): Online-Befragung. In: K. Umlauf; S. Fühles-Ubach; M. Seadle, (Hrsg.): Handbuch Methoden der Bibliotheks- und Informationswissenschaft. Berlin: De Gruyter Saur, 114-127.

Gläser, C.; Kobsch, L. (2020): Student Experience in der Lernwelt Hochschule. Studierende im Fokus der Fallstudien. In: A. Becker; R. Stang (Hrsg.): Lernwelt Hochschule. Dimensionen eines Bildungsbereichs im Umbruch. Berlin; Boston: De Gruyter Saur, 151-170.

Gläser, J.; Laudel, G. (2006): Experteninterviews und qualitative Inhaltsanalyse als Instrumente rekonstruierender Untersuchungen. 2. Aufl., Wiesbaden: VS Verlag für Sozialwissenschaften. 
Hartmann, B. (2016): Kommunikationsmanagement von Clusterorganisationen: theoretische Verortung und empirische Bestandsaufnahme. Wiesbaden: Springer.

Helfferich, C. (2011): Die Qualität qualitativer Daten. Manual für die Durchführung qualitativer Interviews. 4. Aufl. Wiesbaden: VS Verlag für Sozialwissenschaften.

Helfferich, C. (2014): Leitfaden- und Experteninterviews. In: N. Baur, J. Blasius (Hrsg.): Handbuch Methoden der empirischen Sozialforschung. Wiesbaden: Springer, 559-574.

JISC (2015): Learning Space Guide. https://www.jisc.ac.uk/guides/learning-spaces.

Kluge, S. (1999): Empirisch begründete Typenbildung. Zur Konstruktion von Typen und Typologien in der qualitativen Sozialforschung. Opladen: Leske+Budrich.

Knutzen, S.; Brose, A.; Ladwig, T. (2016): Struktur- und Kulturwandel. Lehrinnovative Veränderung an der Technischen Universität Hamburg-Harburg. In: T. Brahm; T. Jenert; D. Euler: (Hrsg.): Pädagogische Hochschulentwicklung. Struktur- und Kulturwandel. Lehrinnovative Veränderung an der Technischen Universität Hamburg-Harburg. Von der Programmatik zur Implementierung. Wiesbaden: Springer, 281-295.

Krueger, R. A.; Casey, M. A. (2009): Focus groups. A practical guide for applied research. 4. Aufl. Los Angeles: Sage.

Kruse, J. (2012): Qualitative Interviewforschung in und mit Fremdsprachen. Eine Einführung in Theorie und Praxis. Weinheim; Basel: Beltz Juventa.

Kruse, J. (2015): Qualitative Interviewforschung. Ein integrativer Ansatz. 2. Aufl. Weinheim; Basel: Beltz Juventa.

Kuckartz, U. (2007): Einführung in die computergestützte Analyse qualitativer Daten. 2. Aufl., Wiesbaden: VS Verlag für Sozialwissenschaften.

Kuckartz, U. (2016): Qualitative Inhaltsanalyse. Methoden, Praxis, Computerunterstützung. 3., überarb. Aufl. Weinheim; Basel: Beltz Juventa.

Kühne, O. (2013): Landschaftstheorie und Landschaftspraxis. Eine Einführung aus sozialkonstruktivistischer Perspektive. Wiesbaden: Springer.

Kultusministerkonferenz (2019): Grundlegende rechtliche Regelungen zu Hochschulen und anderen Einrichtungen des Tertiären Bereichs in der Bundesrepublik Deutschland. https:// www.kmk.org/dokumentation-statistik/rechtsvorschriften-lehrplaene/uebersicht-hochschulgesetze.html.

Lamnek, S. (2005): Qualitative Sozialforschung. Lehrbuch. 4. Aufl., Weinheim: Beltz.

Lamnek, S.; Krell, C. (2016): Qualitative Sozialforschung. Mit Online-Material. 6. Aufl. Weinheim; Basel: Beltz.

Luckmann, T. (2006): Die kommunikative Konstruktion der Wirklichkeit. Wiesbaden: Springer.

Mayring, P. (2009): Qualitative Inhaltsanalyse. In: U. Flick; E. von Kardorff; I. Steinke (Hrsg.): Qualitative Forschung. Ein Handbuch. Reinbek bei Hamburg: Rowohlt, 468-475.

Mayring, P. (2015): Qualitative Inhaltanalyse. Grundlagen und Techniken. 12. Aufl. Weinheim; Basel: Beltz.

Meyen, M.; Löblich, M.; Pfaff-Rüdiger, S.; Riesmeyer, C. (2019): Qualitative Forschung in der Kommunikationswissenschaft. Wiesbaden: Springer.

Miebach, B. (2010): Soziologische Handlungstheorie: Eine Einführung. Wiesbaden: Springer.

NMC - New Media Consortium (2018): Horizon Report Preview 2018. Higher Education Edition. https://library.educause.edu/ /media/files/library/2018/4/previewhr2018.pdf.

P21 (2018): Framework for 21st Century Learning. http://www.p21.org/our-work/p21-framework.

Petersen, T. (2014): Der Fragebogen in der Sozialforschung. Konstanz: UTB. 
Peirce, C. S. (1960): The collected Papers. In: C. Hartshorne; P. Weiss (Hrsg.): Collected Papers of Charles Sanders Peirce. Cambridge M. A.: Harvard University Press.

Radcliffe, D, (2009): A Pedagogy-Space-Technology (PST) Framework for Designing and Evaluating Learning Places. In: D. Radcliffe; H. Wilson; D. Powell; B. Tibbet (Hrsg.): Learning Spaces in Higher Education. Positive Outcomes by Design. St. Lucia/Australia: University of Queensland, 11-16.

Rädiker, S.; Kuckartz, U. (2019): Analyse qualitativer Daten mit MAXQDA. Wiesbaden: Springer VS.

Reinhold, G. (1991): Soziologielexikon. München: Oldenbourg.

Schittenhelm, K. (2017): Mehrsprachigkeit als methodische Herausforderung in transnationalen Forschungskontexten. Zeitschrift für qualitative Forschung 18/1, 101-115.

Scholl, A. (2013): Reaktivität im Forschungsprozess. In: W. Möhring; D. Schlütz (Hrsg.): Handbuch standardisierte Erhebungsverfahren in der Kommunikationswissenschaft. Wiesbaden: Springer, 79-99.

Schnell, R.; Hill, P.; Esser, E. (2011): Methoden der empirischen Sozialforschung. München: Oldenbourg.

Schütz, A.; Luckmann, T. (2003): Strukturen der Lebenswelt. Konstanz: UTB.

Stang, R.; Becker, A. (Hrsg.) (2020): Zukunft Lernwelt Hochschule. Perspektiven und Optionen für eine Neuausrichtung. Berlin; Boston: De Gruyter Saur.

Statistisches Bundesamt (Destatis) (2019): Bildung und Kultur. Finanzen der Hochschulen. Wissen. nutzen. Fachserie 11 Reihe 4.5. https://www.destatis.de/DE/Themen/Gesellschaft-Umwelt/Bildung-Forschung-Kultur/Bildungsfinanzen-Ausbildungsfoerderung/Publikationen/Downloads-Bildungsfinanzen/finanzen-hochschulen-2110450177004.pdf? blob=publicationFile \&v=2.

Tourangeau, R.; Rips, L. J.; Rasinski, K. (2000): The Psychology of Survey Response. Cambridge: Cambridge University Press.

UCISA - Universities and Colleges Information Systems Association (2016): The UK Higher Education Learning Space Toolkit. http://www.ucisa.ac.uk/learningspace.

University of Edinburgh (2013): Interview Consent Form. https://www.ed.ac.uk/files/imports/ fileManager/Interview_Consent_Form.pdf.

Wagner, P.; Hering, L. (2014): Online-Befragung. In: N. Baur; J. Blasius (Hrsg.): Handbuch Methoden der empirischen Sozialforschung. Wiesbaden: Springer, 661-673.

Weichert, H. (2020a): Dimensionen gesetzlicher Rahmungen. Perspektiven deutscher Hochschulgesetzgebung. In: A. Becker; R. Stang (Hrsg.): Lernwelt Hochschule. Dimensionen eines Bildungsbereichs im Umbruch. Berlin; Boston: De Gruyter Saur, 47-58.

Weichert, H. (2020b): Strukturentwicklungspläne und Leitbilder. Orientierungen für strategische Planungen. In: A. Becker; R. Stang (Hrsg.): Lernwelt Hochschule. Dimensionen eines Bildungsbereichs im Umbruch. Berlin; Boston: De Gruyter Saur, 59-70.

Weichert, H.; Stang, R. (2020): Der Blick von außen. Einschätzungen internationaler Expertinnen und Experten. In: A. Becker; R. Stang (Hrsg.): Lernwelt Hochschule. Dimensionen eines Bildungsbereichs im Umbruch. Berlin; Boston: De Gruyter Saur, 171-182.

Werner, P. (2013): Qualitative Befragungen. In: K. Umlauf, S. Fühles-Ubach, M. Seadle (Hrsg.): Handbuch Methoden der Bibliotheks- und Informationswissenschaft. Berlin und Boston: De Gruyter Saur, 128-151.

Wildt, J., Wildt, B. (2011): Lernprozessorientiertes Prüfen im „Constructive Alignment“. In: B. Berendt; H.-P. Voss; J. Wildt (Hrsg.): Neues Handbuch Hochschullehre. Lehren und Lernen 
effizient gestalten. [Teil H.] Prüfungen und Leistungskontrollen. Weiterentwicklung des Prüfungssystems in der Konsequenz des Bologna-Prozesses. Berlin: Raabe.

Zerr, K. (2003): Online Marktforschung. Erscheinungsformen und Nutzenpotentiale. In: A. Theobald; M. Dreyer; T. Starsetzki (Hrsg.): Online-Marktforschung. Theoretische Grundlagen und praktische Erfahrungen. Wiesbaden: Gabler, 7-26.

Zindler, A.; Pohl, A. (2015): Praktische Herausforderungen der Datenerhebung. In: H. Biehl; H. Schoen (Hrsg.): Sicherheitspolitik und Streitkräfte im Urteil der Bürger. Theorien, Methoden, Befunde. Wiesbaden: Springer, 295-322. 